\title{
Minimizing discordances in automated classification of fractionated electrograms in human persistent atrial fibrillation
}

\author{
Tiago P. Almeida ${ }^{1}$ Gavin S. Chu ${ }^{2,3}$ João L. Salinet ${ }^{1,4} \cdot$ Frederique J. Vanheusden $^{1}$. \\ Xin $\mathrm{Li}^{1} \cdot$ Jiun H. Tuan ${ }^{3} \cdot$ Peter J. Stafford ${ }^{3} \cdot$ G. André $\mathrm{Ng}^{2,3,5}$. \\ Fernando S. Schlindwein ${ }^{1,5}$
}

Received: 10 March 2015 / Accepted: 29 January 2016 / Published online: 25 February 2016

(C) The Author(s) 2016. This article is published with open access at Springerlink.com

\begin{abstract}
Ablation of persistent atrial fibrillation (persAF) targeting complex fractionated atrial electrograms (CFAEs) detected by automated algorithms has produced conflicting outcomes in previous electrophysiological studies. We hypothesize that the differences in these algorithms could lead to discordant CFAE classifications by the available mapping systems, giving rise to potential disparities in CFAE-guided ablation. This study reports the results of a head-to-head comparison of CFAE detection performed by NavX (St. Jude Medical) versus CARTO (Biosense Webster) on the same bipolar electrogram data (797 electrograms) from 18 persAF patients. We propose revised thresholds for both primary and complementary indices to minimize the differences in CFAE classification performed by either system. Using the default thresholds [NavX: CFEMean $\leq 120$ ms; CARTO: ICL $\geq 7$ ], NavX classified $70 \%$ of the electrograms as CFAEs, while CARTO detected
\end{abstract}

Electronic supplementary material The online version of this article (doi:10.1007/s11517-016-1456-2) contains supplementary material, which is available to authorized users.

Fernando S. Schlindwein

fss1@leicester.ac.uk; f.s.schlindwein@leicester.ac.uk

1 Department of Engineering, University of Leicester, University Road, Leicester LE1 7RH, UK

2 Department of Cardiovascular Science, University of Leicester, Leicester, UK

3 University Hospitals of Leicester NHS Trust, Leicester, UK

4 Biomedical Engineering, Engineering, Modelling and Applied Social Sciences Centre, Federal ABC University, Santo André, Brazil

5 National Institute for Health Research Leicester Cardiovascular Biomedical Research Unit, Glenfield Hospital, Leicester, UK
$36 \%$ (Cohen's kappa $\kappa \approx 0.3, P<0.0001$ ). Using revised thresholds found using receiver operating characteristic curves [NavX: CFE-Mean $\leq 84 \mathrm{~ms}$, CFE-SD $\leq 47 \mathrm{~ms}$; CARTO: $\mathrm{ICL} \geq 4, \mathrm{ACI} \leq 82 \mathrm{~ms}, \mathrm{SCI} \leq 58 \mathrm{~ms}], \mathrm{NavX}$ classified $45 \%$, while CARTO detected $42 \%(\kappa \approx 0.5$, $P<0.0001)$. Our results show that CFAE target identification is dependent on the system and thresholds used by the electrophysiological study. The thresholds found in this work counterbalance the differences in automated CFAE classification performed by each system. This could facilitate comparisons of CFAE ablation outcomes guided by either NavX or CARTO in future works.

Keywords Atrial fibrillation · Substrate $\cdot$ Mapping · Electrogram fractionation $\cdot$ CFAE $\cdot$ Statistical classification

\section{Introduction}

Atrial fibrillation (AF) is the most common sustained arrhythmia in clinical practice and a leading cause of hospitalization and cardiovascular complications, particularly stroke. It is defined as a supraventricular tachyarrhythmia characterized by uncoordinated atrial activation with consequent deterioration of atrial mechanical function [4]. AF management consists of anticoagulation, antiarrhythmic drugs, electrical cardioversion, and radiofrequency catheter ablation [4]. The latter has been consolidated as the most accepted percutaneous procedure for AF treatment, achieving success rate as high as $90 \%$ in patients with paroxysmal AF [4, 9]. Ablation is still suboptimal in patients with persistent or permanent atrial fibrillation (persAF) due to an incomplete understanding of the mechanistic interaction between relevant atrial substrate and the initiation and maintenance of AF. 
Sustained AF causes changes in the cardiac tissue characteristics, inducing structural and electric remodeling [6]. These regions can potentially host tissue with slow or inhomogeneous conduction, inducing reentry circuits, resulting in fractionated fibrillatory conduction [2], and are important in triggering and perpetuating atrial arrhythmias. Atrial electrograms (AEGs) acquired from such atrial substrate regions demonstrate the low amplitude, multiple deflection activations that characterize fractionated activity (Fig. 1). The ablation of atrial substrate hosting complex fractionated atrial electrograms (CFAEs) has been accepted by many as a useful additional therapy for persAF treatment [4]. Disparities in CFAE-guided ablation outcomes have, however, cast doubt on the efficacy of this approach (Table 1) [7, 14, 16, 19-21, 30, 31].

Automated CFAE detection can be performed during electrophysiological studies by algorithms embedded in commercial three-dimensional electroanatomical mapping (3D EAM) systems [15, 31]. The two EAM systems being used in clinical practice for CFAE mapping are the
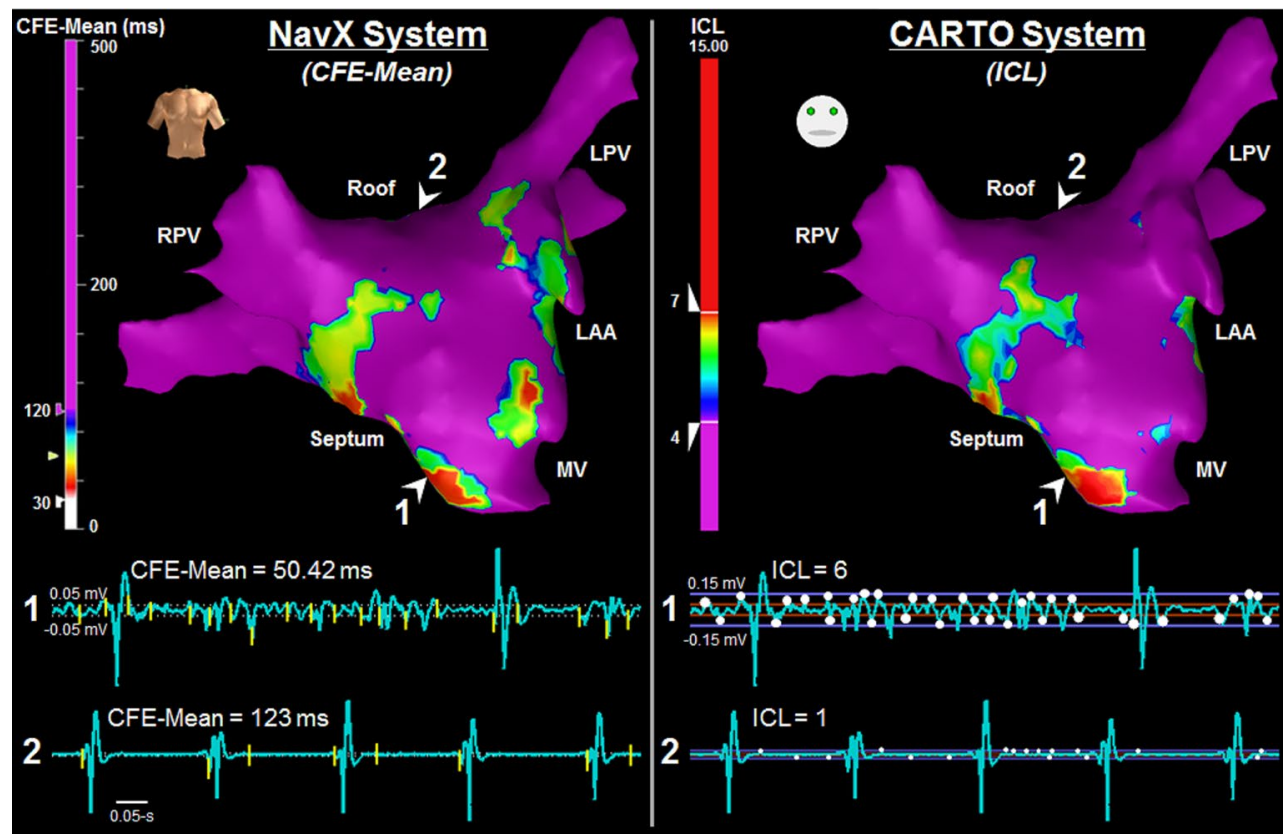

Fig. 1 NavX (left) and CARTO (right) 3D atrial geometry representation for the same patient, with their respective automated CFAE detection algorithms. On the bottom part of the figure, the top traces refer to a segment of fractionated bipolar AEG (AEG 1), and bottom traces refer to a non-fractionated segment of bipolar AEG (AEG 2), both recorded from the LA endocardium. The AEG 1 has CFE-Mean $=50.42 \mathrm{~ms}$ and $\mathrm{ICL}=6$. The AEG 2 has CFE-
Mean $=123 \mathrm{~ms}$ and $\mathrm{ICL}=1$. Explanation regarding the algorithms is provided in the text. $A E G$ atrial electrogram, $C F E$-Mean index used by NavX to quantify AEG fractionation, $F I$ fractionated interval, ICL interval confidence level: index used by CARTO to quantify AEG, $L P V$ left pulmonary veins, $M V$ mitral valve, $R P V$ right pulmonary veins. These abbreviations were used in the subsequent figures
Table 1 Review of CFAE mapping systems, EGM settings, and success rate in different clinical studies

\begin{tabular}{llllll}
\hline Study & \multicolumn{2}{l}{ EGM settings } & Mapping system & No. patients & Success rate (\%) \\
\cline { 2 - 4 } & Amplitude (mV) & Time (ms) & & \\
\hline Elayi et al. [7] & - & $\leq 120$ & NavX & 49 & 61 \\
Lin et al. [14] & - & $\leq 50$ & NavX & 30 & 53 \\
Nademanee et al. [16] & $\leq 0.15$ & $\leq 120$ & CARTO & 121 & 95 \\
Oral et al. [19] & - & $\leq 120$ & CARTO & 100 & 16 \\
Oral et al. [20] & - & $\leq 120$ & CARTO & 50 & 18 \\
Porter et al. [21] & $0.05-0.15$ & $60-120$ & CARTO & 67 & 20 \\
Verma et al. [30] & - & $40-120$ & NavX & 30 & 14 \\
Verma et al. [31] & $0.05 \leq$ & $30-120$ & NavX & 35 & 54 \\
\hline
\end{tabular}

Previous studies conducted either additional or lone CFAE-guided ablation using different mapping systems and varying operator-defined settings, resulting in conflicting outcomes 
NavX ${ }^{\text {TM }}$ (St. Jude Medical, St. Paul, Minnesota) [31] and the CARTO (Biosense Webster, Diamond Bar, California) (Fig. 1) [15]. The algorithms embedded in those systems incorporate CFAE characteristics as initially described by Nademanee et al. [16]. Each algorithm, however, considers different premises to quantify fractionation, and the classification by the different systems does not always agree [13]. We hypothesized that the discordances between systems might result in different ablation target identification and generate discordant clinical results. In this study, we report a direct comparison of the automated CFAE classification performed by the algorithms embedded in NavX and CARTO. We also propose new thresholds for both primary and complementary indices to minimize the differences in CFAE classification performed by either system.

\section{Materials and methods}

\subsection{Automated CFAE detection}

NavX and CARTO provide primary indices to assess CFAE objectively, and complementary indices to further inform the electrophysiological study. Previous works have attempted to optimize CFAE detection using only the primary indices provided by the EAM systems [1]. There are currently no defined thresholds for the complementary indices to characterize CFAEs. Additionally, both systems allow for operator-defined settings-in this work referred to as "EGM settings" - to tune CFAE detection. Previous studies had attempted to optimize CFAE detection using these algorithms by varying EGM settings with different ablation outcomes (Table 1) [7, 14, 16, 19-21, 30, 31].

\subsubsection{The NavX algorithm (EnSite System Version 8.0 Software, 2008)}

NavX provides 3D EAM (Fig. 1 left) and online automated CFAE detection based on CFE-Mean. CFE-Mean is defined as the average fractionated interval (FI) between consecutive negative deflections $(-\mathrm{dV} / \mathrm{dt})$ inside a time window set by the user (from 1 to 8 -s) of sequentially recorded bipolar AEGs (Fig. 1 bottom left) [31]. The negative deflections must meet three criteria in order to be marked: (1) exceed a peak-to-peak threshold greater than baseline noise; (2) have time duration within a threshold to avoid detection of ventricular far-field events; and (3) exceed a refractory period after the previous marked deflection to minimize multiple detections on a single deflection. NavX's default EGM settings include a peak-to-peak sensitivity of $0.05 \mathrm{mV}$, deflection duration of less than $10 \mathrm{~ms}$, and refractory period of $30 \mathrm{~ms}$. AEGs with CFE-Mean within the range of 30-120 ms are considered to be fractionated [31].
These settings can be changed by the user (Table 1) [31]. NavX also computes the standard deviation of FI distribution inside a predefined time window as a complementary index, known as CFE-SD.

\subsubsection{The CARTO algorithm (CARTO 3 System, 2008- 2014, Version 4.3)}

CARTO provides 3D EAM (Fig. 1 right) and online automated CFAE detection based on complex intervals occurring inside a 2.5 -s window of sequentially recorded bipolar AEGs [15]. The algorithm identifies voltage peaks and troughs of bipolar AEGs that exceed a lower voltage threshold - to exclude noise-but do not exceed an upper voltage threshold. Similar to the NavX algorithm, the user can alter these CARTO thresholds (Table 1). The time intervals between successive peaks and troughs occurring within the voltage window are automatically marked by the system. The complex intervals marked within a time interval duration-defined by the operator-are identified during a 2.5 -s time window (Fig. 1 bottom right). The number of identified complex intervals is referred to as the interval confidence level (ICL) and characterizes the repetitiveness of the CFAE complexes. CARTO's default EGM settings consider a voltage window of $0.05-0.15 \mathrm{mV}$ and a programmable time interval of 50-110 ms. Typically, ICL $<4$ represents low fractionation, $4 \leq \mathrm{ICL}<7$ refers to moderate fractionation, and ICL $\geq 7$ indicates high fractionation [15]. CARTO software also finds, as complementary indices, the average of the identified interval, referred to as the average complex interval (ACI), and the shortest identified interval, referred to as the shortest complex interval (SCI).

\subsection{Study population}

The study population consisted of 18 persAF patients (16 male; mean age $56.1 \pm 9.3$ years; history of $\mathrm{AF}$ $67.2 \pm 45.6$ months) referred to our institution for firsttime catheter ablation. Details of the clinical characteristics of the study subjects have been provided elsewhere [28]. Study approval was obtained from the local ethics committee, and all procedures were performed with full informed consent.

\subsection{Electrophysiological Study}

Details of the electrophysiological study and mapping procedure have been described elsewhere [28]. Briefly, 3D left atrium (LA) geometry was created within [Ensite] NavX using a deflectable, variable loop circular PV mapping catheter (Inquiry Optima, St. Jude Medical). Pulmonary vein isolation (PVI) was performed, followed by the creation of a single roof line (Cool Path Duo irrigated RF 
catheter, St. Jude Medical). No additional ablation targeting CFAE was performed in this study. Sequential point-bypoint bipolar AEGs were collected from 15 predetermined atrial regions before and after LA ablation with the ablation catheter [28]. A total of 797 AEGs were recorded from the LA, with a sampling frequency of $1.2 \mathrm{kHz}$, and band-pass filtered within 30-300 Hz. When an improvement of signal-to-noise ratio was necessary, a $50-\mathrm{Hz}$ Notch filter was applied.

\subsection{Comparing CFAE definitions between EAM systems}

\subsubsection{Signal analysis}

Each AEG, its corresponding CFE-Mean, and CFE$\mathrm{SD}$ were exported from NavX with three time window lengths $(2.5,5$, and 8-s). A validated off-line MATLAB algorithm was used to compute the ICL, ACI, and SCI of each exported AEG for CFAE identification as defined by CARTO—see Supplemental Materials.

Currently, the CARTO system considers only 2.5-s AEGs for CFAE detection. Hence, there is no validated ICL threshold for CFAE classification using time windows longer than $2.5 \mathrm{~s}$. Nevertheless, the effects of different time windows-2.5, 5, $8 \mathrm{~s}$-were assessed on ICL and CFE-Mean for the completeness of the investigation-see
Supplemental Materials. Little influence on overall CFEMean was found when using different time windows. Therefore, for the remaining parts of the study, NavX and CARTO indices were measured using fixed 2.5-s AEG duration to allow a like-for-like comparison [15, 31].

\subsubsection{Influence of EGM settings on CFAE classification}

CFE-Mean and ICL were individually assessed, exploring the effects of varying EGM settings: NavX EGM settings (30-120 ms) and CARTO EGM settings (50$110 \mathrm{~ms}$ ). Hence, the threshold for CFAE classification was $30-120$ ms if CFE-Mean was measured using NavX EGM settings, and $50-110 \mathrm{~ms}$ for CARTO EGM settings [15, 31]. ICL $\geq 7$ was used as the default threshold for CARTO CFAE categorization to assess the impact of both NavX and CARTO EGM settings [21].

\subsubsection{CFAE detection thresholds for CFE-Mean and ICL}

CFAE detection and classification were performed on 697 randomly sampled AEGs (out of the total 797), first using CFE-Mean and then ICL. This dataset was used to create receiver operating characteristic (ROC) curves and hence obtain the optimum sensitivity and specificity thresholds for both indices, using the counterpart index as the comparator (Fig. 2a) [8]. The ICL-based classification was
Fig. 2 Illustration of the method for training the ROC curves and validating the proposed revised thresholds. a Thirty datasets-with 679 randomly selected AEGs eachwere used to train and create the ROC curves. b For each of the 30 datasets, the remaining 118 AEGs were used to validate the thresholds found in the ROC curves
(A)

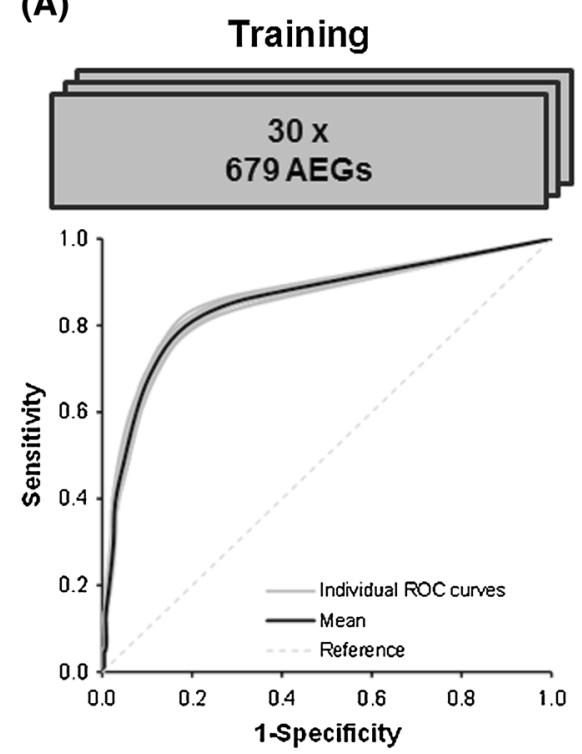

797 AEGs

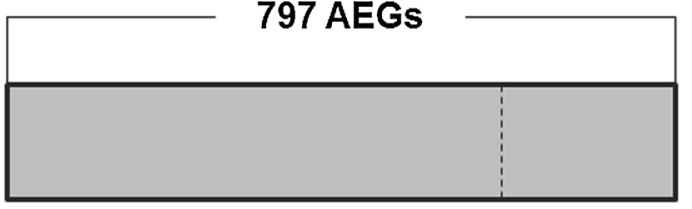

(B)
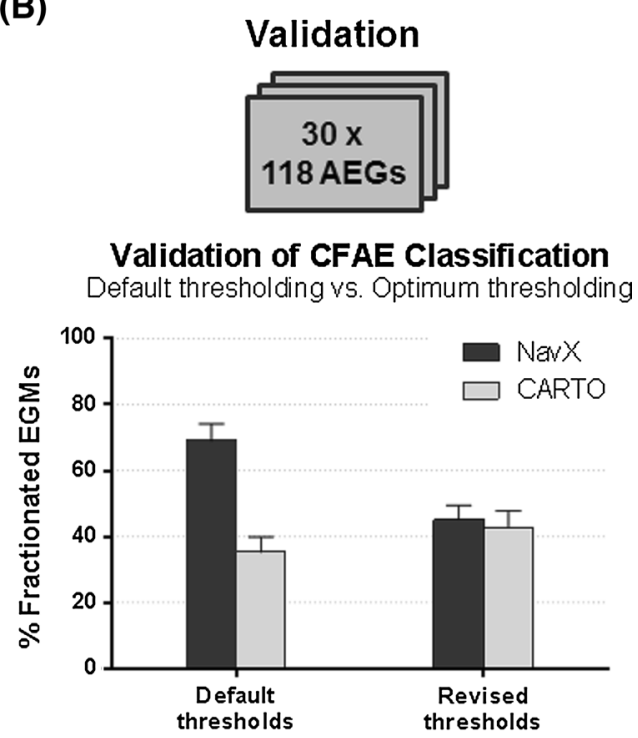
assessed by creating a ROC curve, using CFE-Mean and the NavX EGM settings (CFE-Mean $\leq 120 \mathrm{~ms}$ ) as the reference classification (CFAE/non-CFAE) [31]. The revised threshold for ICL was identified based on the optimum sensitivity and specificity on the ROC curve-defined as the point on the curve with the shortest distance to the top left corner of the graph. Similarly, the CFE-Mean-based classification was assessed by creating a ROC curve, using ICL and the CARTO EGM settings (ICL $\geq 7$ ) as the reference classification (CFAE/non-CFAE) [21]. The revised threshold for CFE-Mean was identified based on the optimum sensitivity and specificity on the ROC curve. Area under the ROC curve (AUROC) and the $P$ value were also calculated.

This process was iterated 30 times, each time with a different dataset of randomly sampled AEGs for ROC curve construction (697 AEGs), giving a total of 30 ROC curves for ICL and 30 for CFE-Mean in order to minimize data sampling and selection biasing.

\subsubsection{CFAE detection thresholds for CFE-SD, ACI, and $S C I$}

The revised thresholds for both CFE-Mean and ICL found in the ROC curves were used to perform a new CFAE classification on the 30 sets of 697 randomly sampled AEGs. In this new classification, an AEG was classified as CFAE only if both CFE-Mean and ICL agreed with the classification using their revised thresholds. These classifications were used to create ROC curves and hence obtain the optimum sensitivity and specificity thresholds for the complementary indices-CFE-SD, ACI, and SCI.

\subsubsection{Validation of the revised thresholds for CFAE detection performed by $\mathrm{NavX}$ and CARTO}

The revised thresholds found in the ROC curves for both NavX-CFE-Mean and CFE-SD - and CARTO-ICL, $\mathrm{ACI}$, and SCI-were validated using the remaining 118 AEGs (30 sets of 118 AEGs randomly selected) (Fig. 2b).

For each of the 30 datasets, CFAE classification was performed using the combined assessment of both primary and complementary indices. Explicitly, an AEG was classified as CFAE if it complied with both CFE-Mean and CFE-SD for NavX classification. Similarly, an AEG was classified as CFAE if it complied with ICL, ACI, and SCI for CARTO classification.

\subsection{Statistical analysis}

All continuous normally distributed variables are expressed as mean \pm standard deviation (SD). Continuous non-normally distributed variables are expressed as median \pm interquartile interval. Nonparametric paired multiple data were analyzed using the Friedman test with Dunn's correction, while nonparametric unpaired data were analyzed using the Mann-Whitney test. Categorical data were expressed as percentages and analyzed using the two-sided Yatescorrected Chi-square test. The quantification of the agreement between the rankings made by CFE-Mean and ICLas measured using their default settings-was assessed by the Spearman's correlation between both indices. The level of agreement in the CFAE classification performed by the two systems was assessed by the Cohen's kappa $(\kappa)$ score [5]. Kappa score within range $0 \leq \kappa<0.4$ suggests marginal agreement between two indices; $0.4 \leq \kappa \leq 0.75$ good agreement; and $\kappa>0.75$ excellent agreement [12]. $P$ values less than 0.05 were considered statistically significant.

\section{Results}

\subsection{Influence of EGM settings on CFAE classification}

CFAE classification differed as performed by CFE-Mean and ICL using their respective default EGM settings. CFEMean classified $70 \%$ of the AEGs as CFAEs using NavX EGM settings, while ICL classified $36 \%$ using CARTO EGM settings $(P<0.0001)$.

Changing the EGM settings alters CFAE classification. Figure 3 demonstrates the importance of EGM settings for CFAE classification. It illustrates the 3D CFAE map (anterior and posterior views) of one of the patients according to CFE-Mean and ICL, using different EGM settings. CFE-Mean measured using NavX's settings identified more atrial regions as CFAEs, while ICL measured using CARTO's settings showed fewer regions as fractionated. When analyzing the entire database, the NavX EGM settings consistently categorized more AEGs as fractionated than the CARTO EGM settings (70 vs. $54 \%, P<0.0001$ for CFEMean; 62 vs. $36 \%, P<0.0001$ for ICL).

\subsection{CFAE detection thresholds for CFE-Mean and ICL}

The comparison between CFE-Mean and ICL (measured using their default settings) for each of the 797 AEGs is illustrated in Fig. 4a. The respective default thresholds for both CFAE detection techniques are highlighted. The four quadrants delimited by the thresholds illustrate the zones of agreement and disagreement between CFE-Mean and ICL. Spearman's correlation between the classifications by the two indices was $\rho=-0.563(P<0.0001) .230$ (out of 797) AEGs with organized activations were found in the non-fractionated agreement zone (green). When looking at the AEGs corresponding to the disagreement quadrants (gray), 282 AEGs have been classified as CFAEs by NavX but not by CARTO in one gray region (bottom left). In the other gray 


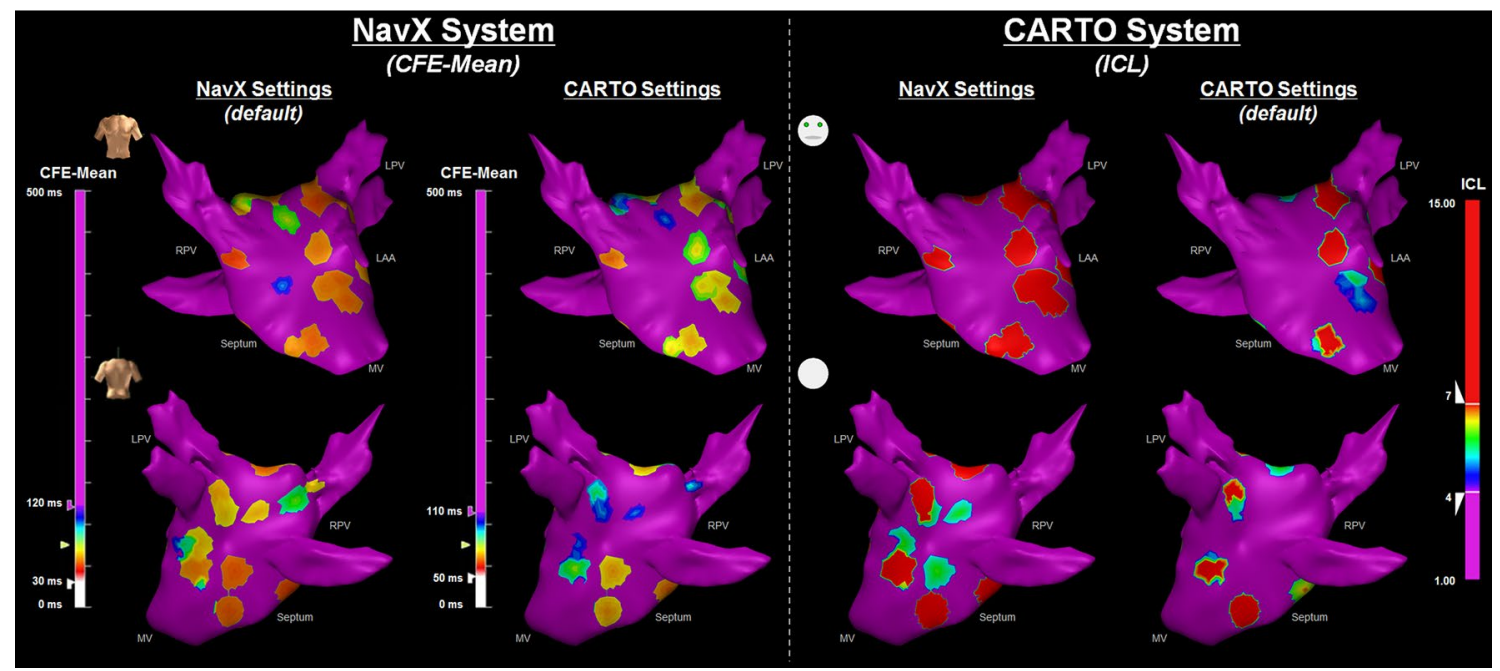

Fig. 3 3D CFAE map showing the anterior (upper) and posterior (bottom) view of the LA from one patient according to CFE-Mean (left-hand side) and ICL (right-hand side), using different EGM set- tings. NavX EGM settings identify more regions of the LA as CFAE than CARTO's when applied to either CFE-Mean or ICL
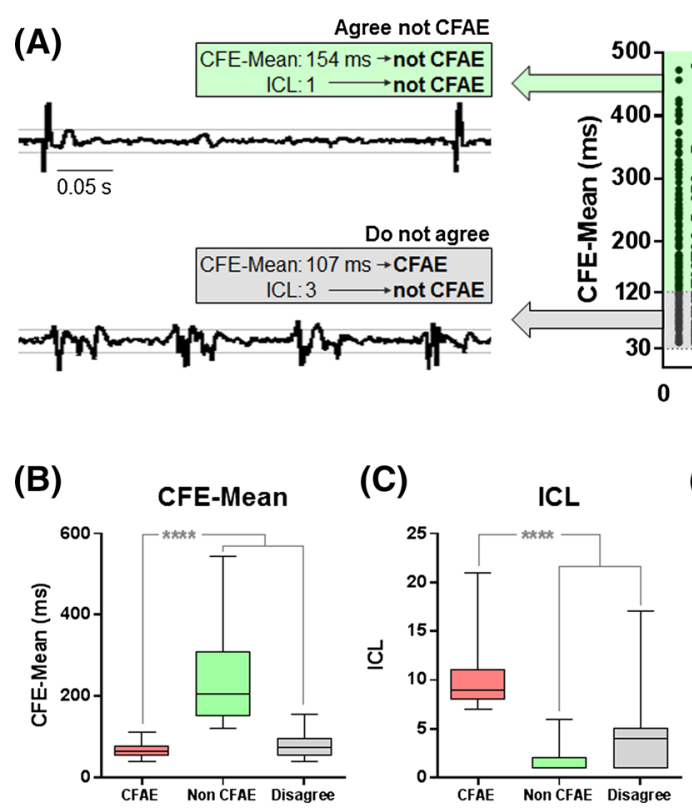

(C)
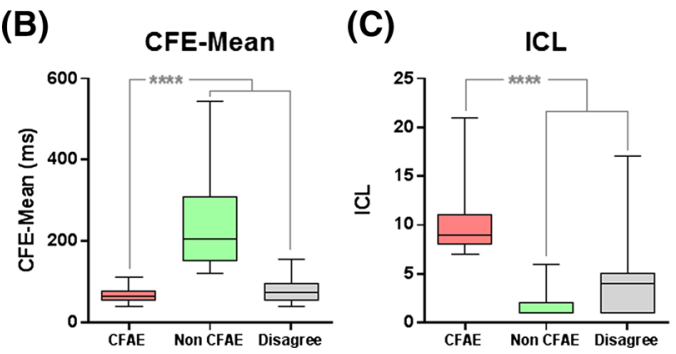

(D)

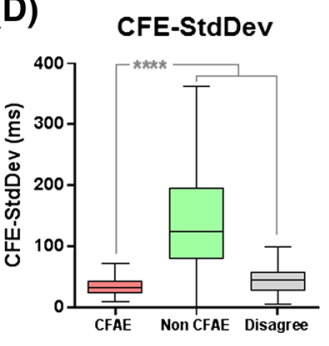

(E)

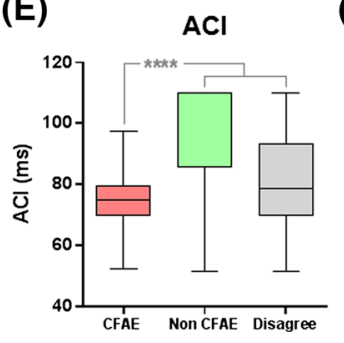

(F)
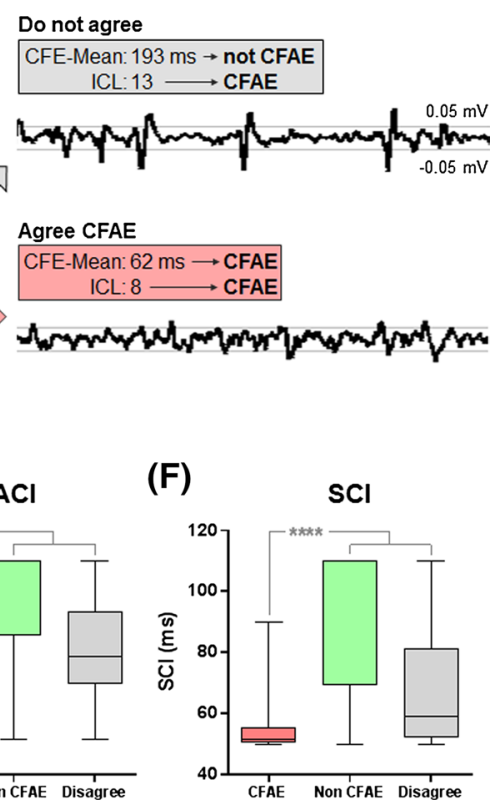

Fig. 4 a Comparison between CFE-Mean and ICL measured for all 797 AEGs, as determined by default NavX and CARTO EGM settings, respectively. Their respective default thresholds are highlighted (CFE-Mean $\leq 120 \mathrm{~ms}$; ICL $\geq 7$ ). Four quadrants were delimited: two quadrants where ICL and CFE-Mean agreed in terms of categorization, i.e., whether an AEG is fractionated or not fractionated, and two quadrants in which they disagreed. Examples of AEGs for each of the quadrants are shown, to illustrate the characteristics of each group. 230 (out of 797) AEGs with organized activations were found in the non-fractionated agreement zone (green). When looking at the AEGs

corresponding to the disagreement quadrants (gray), one notices that they are less organized, still with distinguishable activations. In one gray region (bottom left), 282 AEGs have been classified as CFAEs by NavX but not by CARTO. In the other gray region (top right), 12 AEGs have been classified as CFAEs by CARTO, but not by NavX. Finally, 273 highly fractionated AEGs were found in the CFAE agreement zone (red). The distributions for the AEGs classified as nonCFAEs or CFAEs by both systems, as well as AEGs that had different classifications for each system, are shown according to CFE-Mean (b), ICL (c), CFE-SD (d), ACI (e), and SCI (f). $* * * * P<0.0001$ 
region (top right), 12 AEGs have been classified as CFAEs by CARTO, but not by NavX. Finally, 273 highly fractionated AEGs were found in the CFAE agreement zone (red).

Quantitative results are provided in Fig. $4 \mathrm{~b}-\mathrm{f}$ to characterize the AEGs after the objective comparison between CFE-Mean and ICL. The results show the distributions for primary and complementary indices (CFE-Mean, ICL, CFE-SD, ACI, and SCI, respectively) for the AEGs classified as non-CFAEs or CFAEs by both systems, as well as AEGs that had different classifications for each system. For all indices, the distributions for the AEGs classified as CFAE (red) were significantly different $(P>0.0001)$ than the combined distributions of non-CFAEs together with those AEGs with different classifications for each system (green + gray)

Figure $5 \mathrm{a}, \mathrm{b}$ shows the ROC curves according to the CFAE classification performed by CFE-Mean and ICL. Table 2 provides the sensitivity, specificity, and AUROC values for each case. The details of the ROC curves from the 30 datasets are provided in the Supplemental Materials.

The ICL-based classification suggests that the default threshold for CARTO (ICL $\geq 7$ ) provides high specificity but poor sensitivity for CFAE detection (Fig. 5a; Table 2A). The revised threshold found from the ROC curves (ICL $\geq 3.8 \pm 0.4$ ) provides optimum sensitivity and specificity for CFAE detection and classification using CFE-Mean $\leq 120 \mathrm{~ms}$ as the reference classification.

The CFE-Mean-based classification suggests that the default threshold for NavX (CFE-Mean $\leq 120 \mathrm{~ms}$ ) provides high sensitivity but poor specificity for CFAE detection (Fig. 5b; Table 2B). The revised threshold found from the ROC curves (CFE-Mean $\leq 84 \pm 0.4 \mathrm{~ms}$ ) provides optimum sensitivity and specificity for CFAE detection and classification using ICL $\geq 7$ as the reference classification.

Other thresholds for CFE-Mean- and ICL-based classification have been explored and support the present findings. Details are provided in the Supplemental Materials.

\subsection{CFAE detection thresholds for CFE-SD, ACI, and SCI}

Figure 5c-e shows the ROC curves for CFE-SD, ACI, and SCI according to the CFAE classification performed by both CFE-Mean and ICL, using their revised thresholds. Table 3 provides the sensitivity, specificity, and AUROC values. The details of the ROC curves from the 30 datasets are also provided in the Supplemental Materials.

The ROC curves suggest that CFE-SD $\leq 46.6 \pm 0.8 \mathrm{~ms}$ (Fig. 5c), ACI $\leq 82.2 \pm 0.3 \mathrm{~ms}$ (Fig. 5d), and $\mathrm{SCI} \leq 58.6 \pm 0.4 \mathrm{~ms}$ (Fig. $5 \mathrm{e}$ ) provide optimum sensitivity and specificity for CFAE detection, when considering the agreement between CFE-Mean and ICL for CFAE classification.

\subsection{Validation of the revised thresholds for CFAE detection performed by NavX and CARTO}

Using the default thresholds (NavX: CFE-Mean $\leq 120 \mathrm{~ms}$; CARTO: ICL $\geq 7$ ), NavX classified $69 \pm 5 \%$ of the AEGs from the internal validation datasets as CFAEs, while CARTO detected $35 \pm 5 \%(P<0.0001)$. With the revised thresholds (NavX: CFE-Mean $\leq 84.1 \pm 0.4 \mathrm{~ms}$ and CFE-SD $\leq 46.6 \pm 0.8 \mathrm{~ms}$; CARTO: ICL $\geq 3.8 \pm 0.4$, $\mathrm{ACI} \leq 82.2 \pm 0.3 \mathrm{~ms}$ and $\mathrm{SCI} \leq 58.6 \pm 0.4 \mathrm{~ms})$, NavX classified $45 \pm 4 \%$, while CARTO detected $42 \pm 5 \%$ $(P<0.0001)$. These results are illustrated in Fig. $5 f$.

Figure $5 \mathrm{~g}$ illustrates CFAE classification performed by NavX and CARTO using the default (left-hand side) and revised (right-hand side) thresholds for the same patient. The CFAE maps produced by both systems using their default thresholds are very discordant, and these differences were minimized when each system used their revised thresholds. The CFAE maps created by both systems using their revised thresholds identified more similar atrial regions as target for ablation. The Kappa score between the CFAE categorization performed by NavX and CARTO significantly increased $(P<0.0001)$ from $\kappa=0.34 \pm 0.07$ (marginal agreement, $P<0.0001$ ) using their default thresholds to $\kappa=0.45 \pm 0.10$ (good agreement, $P<0.0001)$ with the proposed revised thresholds.

\section{Discussion}

This is the first study that uses the same bipolar AEG data collected during persAF ablation to compare CFAE detection performed by the algorithms embedded in NavX and CARTO systems. Additionally, the thresholds for the indices used by both systems were adjusted to minimize the differences between them. The results presented here highlight the discordances in CFAE classification between both systems, which could produce potential disparities in CFAE-guided ablation. The proposed revised thresholds counterbalance the differences in automated CFAE classification performed by the algorithms embedded in each system and reduce the discordances between them. Unifying methods of CFAE classification would allow comparable CFAE maps to be generated which could then act as a standard for future clinical studies.

\subsection{Atrial substrate characterized by CFAE}

The true significance of CFAE in the pathophysiology of $\mathrm{AF}$ remains to be determined. Although it is believed that CFAEs represent atrial substrate during persAF $[2,4,16$, $23,32]$, recent investigations have shown that fractionated AEGs during AF may characterize remote atrial far-field activity $[3,17]$ and passive wavefront collision within the 
(A) Reference classification: CFE-Mean $\leq 120 \mathrm{~ms}$ Classifier being tested: $I C L$

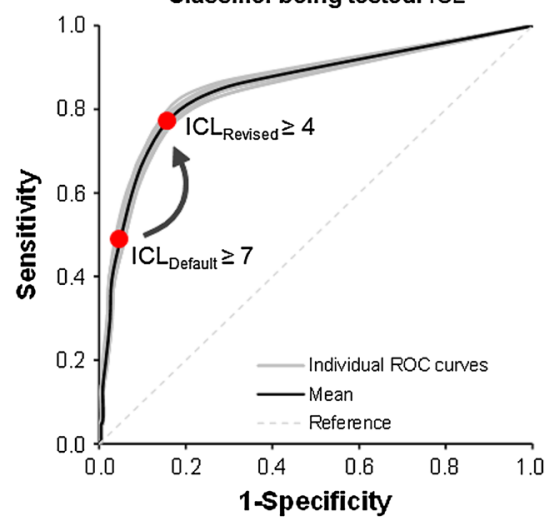

(D) Reference classification: CFE-Mean \& ICL

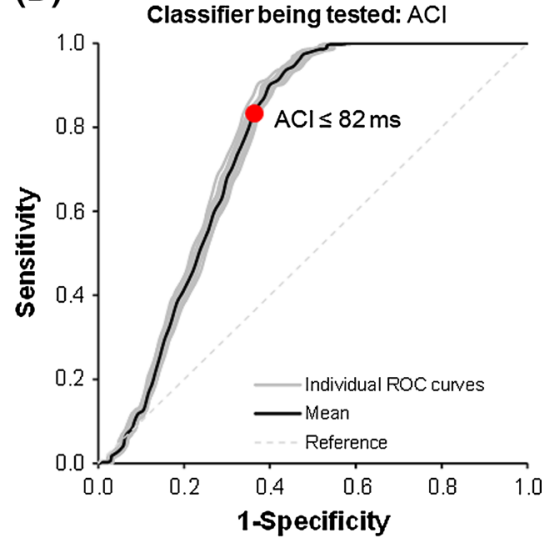

(B) Reference classification: $I C L \geq 7$

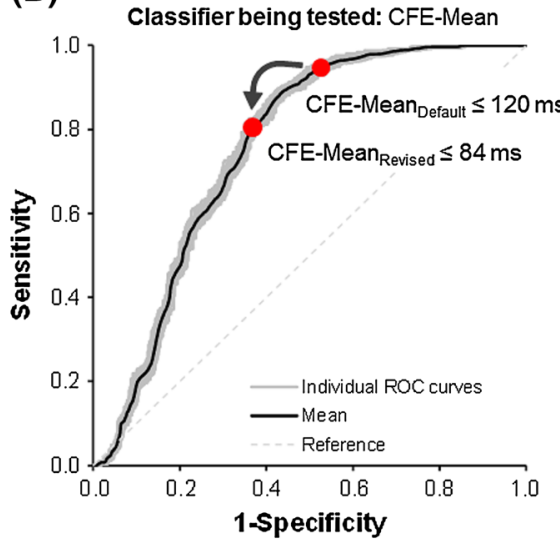

(E) Referenceclassification:CFE-Mean \& ICL

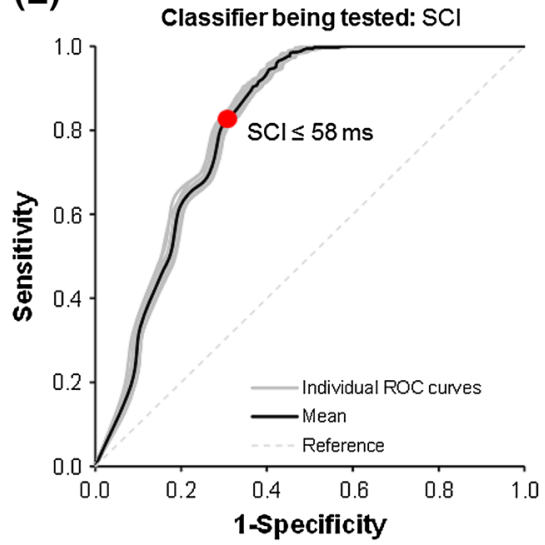

(C) Reference classification: CFE-Mean \& ICL Classifier being tested: $\mathrm{CFE}-\mathrm{StdDev}$

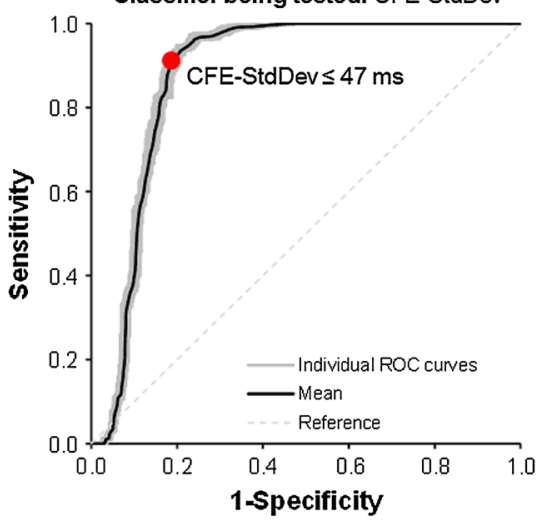

(F) Validation of CFAE Classification Default thresholding vs. revised thresholding

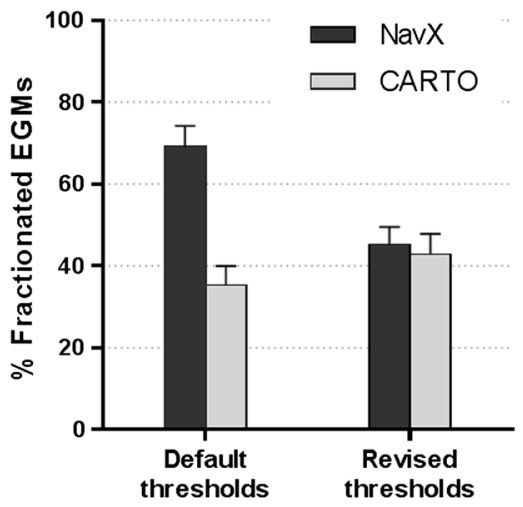

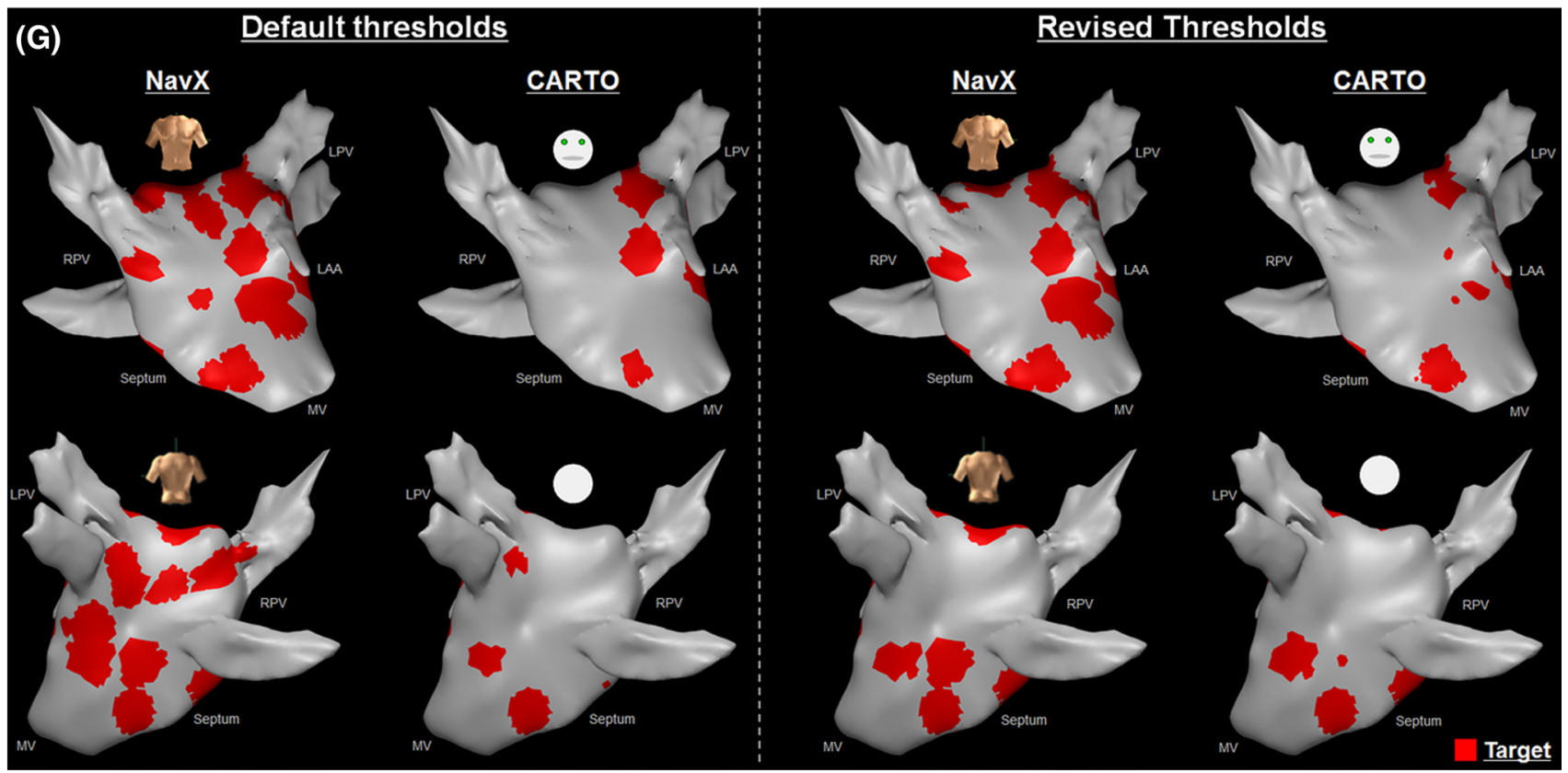

atrial anatomy [24]. Recent work has reported no benefit when ablation of CFAEs was performed in addition to PVI in persAF patients [29]. However, this work has been openly criticized for (1) not considering the combination of PVI, anatomical lines creation and CFAE ablation, and (2) the CFAE mapping algorithm used [26]. Discordances in CFAE mapping algorithms create significant difficulty in comparing CFAE ablation studies. Minimizing 
4 Fig. 5 Mean (black lines) and individual (gray lines) receiver operating characteristic (ROC) curves used to adjust CFAE classification. ROC curve of a ICL-based classification using CFE-Mean $\leq 120 \mathrm{~ms}$ as the reference classification and; $\mathbf{b}$ CFE-Mean-based classification using ICL $\geq 7$ as the reference classification. The CFAE classification in agreement with CFE-Mean and ICL using their revised thresholds (CFE-Mean $\leq 84.1 \pm 0.4 \mathrm{~ms}$; ICL $\geq 3.8 \pm 0.4$ ) was used to create ROC curves of $\mathbf{c}-\mathbf{e}$. $\mathbf{c}$ CFE-SD; $\mathbf{d}$ ACI and; e SCI. The area under the ROC curve (AUROC) and optimum sensitivity and specificity for each measure are listed in Tables 2 and 3. f Validation of the revised thresholds for CFAE classification. g Illustration of CFAE classification performed by NavX and CARTO in the LA from one patient using their default (left) and revised (right) thresholds. Explanations are provided in the text

the differences in CFAE classification between NavX and CARTO may help to clarify the significance of CFAE as a driver of persAF. Therefore, prudence is needed when comparing the outcomes in AF ablation incorporating CFAEtargeted approaches in different electrophysiological studies using different mapping systems [7, 14, 16, 19-21, 29-31].

\subsection{The lack of a gold standard for CFAE definition}

There is currently no gold standard for CFAE classification in human persAF. This remains one of the biggest challenges for CFAE-based ablation. Visual assessment performed by experts could help on arbitration of fractionation, but would also introduce subjectivity to the method as each specialist has his/her own perception of what defines fractionation [1, 10, 18]. Additionally, even if the experts were able to visually identify if an AEG is a CFAE, still this would not be sufficient to effectively conclude that the AEG is a true representation of atrial substrate (these would correspond to atrial regions that organize AF when ablated). This ultimate conclusion is only possible by assessing if AF becomes more organized after ablating this particular atrial region by assessing if there is an appreciable change in the rhythm-either AF termination or increase in $\mathrm{AF}$ cycle length. Any "external reference" other than "AEGs that organized AF after ablation" would only introduce subjectivity to the method and would contribute little to objectively identifying LA regions as a surrogate of proarrhythmogenic sites. Therefore, visual assessment of fractionation for the recorded signals performed by clinicians was not included in this study. The present study focused on the classification performed automatically by both systems as currently performed in CFAE-guided ablation therapy, without the interference and subjectivity induced by operators. This allowed for an objective investigation of CFAE detection by investigating each system-one at a time-as being the "gold" standard for CFAE classification.

\subsection{CFAE detection performed by NavX and CARTO algorithms}

We have shown that CFAE target identification is dependent on the system used and settings applied during the

Table 2 Sensitivity and specificity for A ICL-based classification using CFE-Mean $\leq 120 \mathrm{~ms}$ as the reference classification, and B CFE-Meanbased classification using ICL $\geq 7$ as the reference classification

\begin{tabular}{|c|c|c|c|c|c|c|}
\hline & Reference classifier & Classifier being tested & Sensitivity & 1-Specificity & AUROC & $P$ value \\
\hline \multirow[t]{2}{*}{ A } & \multirow{2}{*}{ CFE-Mean $\leq 120 \mathrm{~ms}$} & $\mathrm{ICL}_{\text {Default }} \geq 7$ & $0.492 \pm 0.008$ & $0.050 \pm 0.005$ & \multirow[t]{2}{*}{$0.852 \pm 0.005$} & \multirow[t]{2}{*}{$* * * *$} \\
\hline & & $\mathrm{ICL}_{\text {Revised }} \geq 3.8 \pm 0.4$ & $0.777 \pm 0.022$ & $0.162 \pm 0.022$ & & \\
\hline \multirow[t]{2}{*}{$\mathrm{B}$} & \multirow[t]{2}{*}{$\mathrm{ICL} \geq 7$} & CFE-Mean $_{\text {Default }} \leq 120 \mathrm{~ms}$ & $0.958 \pm 0.005$ & $0.552 \pm 0.009$ & \multirow[t]{2}{*}{$0.755 \pm 0.005$} & \multirow[t]{2}{*}{$* * * *$} \\
\hline & & CFE-Mean $_{\text {Revised }} \leq 84.1 \pm 0.4 \mathrm{~ms}$ & $0.807 \pm 0.010$ & $0.362 \pm 0.006$ & & \\
\hline
\end{tabular}

Mean $( \pm \mathrm{SD})$ of each sensitivity/specificity point from the 30 receiver operating characteristic (ROC) curves according to the CFAE classification performed by CFE-Mean and ICL. The mean $( \pm$ SD) area under the ROC curve (AUROC) and optimum sensitivity and specificity for each measure are listed

AUROC area under receiver operating characteristic curve, ICL interval confidence level

$* * * * P<0.0001$

Table 3 Sensitivity and specificity for CFE-SD, ACI, and SCI according to CFAE classification agreement between CFE-Mean and ICL

\begin{tabular}{lllll}
\hline Thresholds & Sensitivity & 1-Specificity & AUROC & $P$ value \\
\hline CFE-SD $\leq 46.6 \pm 0.8 \mathrm{~ms}$ & $0.905 \pm 0.012$ & $0.185 \pm 0.008$ & $0.877 \pm 0.014$ & $* * * *$ \\
$\quad$ ACI $\leq 82.2 \pm 0.3 \mathrm{~ms}$ & $0.827 \pm 0.010$ & $0.360 \pm 0.009$ & $0.759 \pm 0.006$ & $* * * *$ \\
SCI $\leq 58.6 \pm 0.4 \mathrm{~ms}$ & $0.816 \pm 0.012$ & $0.300 \pm 0.009$ & $0.812 \pm 0.005$ & $* * * *$ \\
\hline
\end{tabular}

Mean ( \pm SD) of each sensitivity/specificity point from the 30 receiver operating characteristic (ROC) curves according to the CFAE classification performed concurrently by both CFE-Mean and ICL. The mean $( \pm \mathrm{SD})$ area under the ROC curve (AUROC) and optimum sensitivity and specificity for each measure are listed. $* * * * P<0.0001$ 
procedure. Different CFAE mapping algorithms are based on different premises to measure fractionation. For instance, NavX identifies AEGs with a very short cycle length with or without multiple potentials. CARTO, on the other hand, mostly measures AEGs that are composed of two deflections or more and/or have a perturbation of the baseline with continuous deflections [16].

The comparison between both mapping systems using their default settings produced a low correlation ( $\rho=-0.563, P<0.0001$ ), which supports recently published data [13]. However, the Spearman's correlation does not consider the thresholds for CFAE classification. The values - and correlation - of CFE-Mean and ICL only have a full electrophysiological meaning when linked with the thresholds used. This information is provided by the Kappa score.

It is known that both systems are not used simultaneously during atrial substrate mapping and that physicians frequently vary the settings for CFAE mapping in a patientspecific manner [7, 14, 16, 19-21, 29-31]. Our results propose revised thresholds for CFAE detection to be used independently by NavX and CARTO to even out the discordances between them. Therefore, a CFAE map created with NavX utilizing the revised NavX's thresholds will look more similar to the one that would have been created with CARTO utilizing CARTO's revised thresholds proposed in this work, as illustrated in Fig. 5g.

The use of both primary and complementary indices is an additional way to even out differences in CFAE classification performed by each system. There are little data available about the complementary indices measured by automated algorithms being used to either target or support CFAE identification during atrial substrate mapping $[22,25,27]$. However, the ROC curves generated using the agreement between CFE-Mean and ICL as the reference for the classification of CFE-SD, ACI, and SCI (Fig. 5c-e) provide evidence that these complementary indices can help to distinguish CFAE from non-CFAE effectively. This would further improve the agreement of CFAE classification performed by both systems.

\section{Limitations}

The current study was limited to retrospective data. Further understanding of the underlying cardiac electrophysiological mechanisms behind CFAEs would be helpful for the validation of the suggested revised thresholds, such as in (1) computational intracardiac models that simulate both atrial electrical activity and ablation procedures during $\mathrm{AF}$ [11], and (2) prospective studies using the revised thresholds in the identification of ablation targets during substrate mapping.

\section{Conclusions}

This study provides a direct quantitative comparison of CFAE detection during persAF, applying the automated algorithms embedded in NavX and CARTO systems to the same bipolar AEG data. We have demonstrated that CFAE mapping (and thus ablation target identification) varies significantly for the same individual, depending on the system and its settings. Our work takes a first step to understanding and minimizing the discordance between NavX and CARTO. We propose revised thresholds that adjust sensitivity and specificity of CFAE detection as independently performed by NavX (CFE-Mean $\leq 84 \mathrm{~ms}$; CFE-SD $\leq 47 \mathrm{~ms})$ and CARTO (ICL $\geq 4 ; \mathrm{ACI} \leq 82 \mathrm{~ms}$; $\mathrm{SCI} \leq 58 \mathrm{~ms}$ ). These thresholds counterbalance the intrinsic differences between the CFAE algorithms embedded in each system, allowing comparable CFAE maps to be generated which would facilitate the direct comparison of CFAEguided ablation outcomes in future studies.

Acknowledgments The authors thank Dr. Guilherme L. Pimentel and Dr. Will Nicolson for their valuable suggestions. The research leading to these results was funded by the Leicester NIHR Cardiovascular Biomedical Research Unit, UK. Mr. Almeida and Dr. Salinet have received research Grants from Conselho Nacional de Desenvolvimento Científico e Tecnológico (CNPq, Brazil, Grants Nos. 200251/2012-0; 200598/2009-0, respectively). Dr. Chu has received a research Grant from St. Jude Medical and honoraria from Biosense Webster. Dr. Vanheusden has received a research Grant from the University Hospitals Leicester NIHR Cardiovascular Biomedical Research Unit, UK. Dr. Stafford has received speaker fees from St. Jude Medical and Biosense Webster. Prof. $\mathrm{Ng}$ has received research fellowship from St. Jude Medical and speaker fees and honoraria from Biosense Webster. All other authors have reported that they have no relationships relevant to the contents of this paper to disclose.

Open Access This article is distributed under the terms of the Creative Commons Attribution 4.0 International License (http://creativecommons.org/licenses/by/4.0/), which permits unrestricted use, distribution, and reproduction in any medium, provided you give appropriate credit to the original author(s) and the source, provide a link to the Creative Commons license, and indicate if changes were made.

\section{References}

1. Aizer A, Holmes DS, Garitski AC et al (2008) Standardization and validation of an automated algorithm to identify fractionation as a guide for atrial fibrillation ablation. Heart Rhythm 5:1134-1141

2. Ashihara T, Haraguchi R, Nakazawa K et al (2012) The role of fibroblasts in complex fractionated electrograms during persistent/permanent atrial fibrillation: implications for electrogrambased catheter ablation. Circ Res 110:275-284

3. Atienza F, Calvo D, Almendral J et al (2011) Mechanisms of fractionated electrograms formation in the posterior left atrium during paroxysmal atrial fibrillation in humans. J Am Coll Cardiol 57:1081-1092

4. Calkins H, Kuck KH, Cappato R et al (2012) 2012 HRS/EHRA/ ECAS expert consensus statement on catheter and surgical ablation of atrial fibrillation: recommendations for patient selection, 
procedural techniques, patient management and follow-up, definitions, endpoints, and research trial design. Europace 14:528-606

5. Cohen J (1968) Weighted kappa: nominal scale agreement with provision for scaled disagreement or partial credit. Psychol Bull 70:213-220

6. de Bakker JMT, Wittkampf FHM (2010) the pathophysiologic basis of fractionated and complex electrograms and the impact of recording techniques on their detection and interpretation. Circ Arrhythm Electrophysiol 3:204-213

7. Elayi CS, Verma A, Di Biase L et al (2008) Ablation for longstanding permanent atrial fibrillation: results from a randomized study comparing three different strategies. Heart Rhythm 5:1658-1664

8. Fawcett T (2006) An introduction to ROC analysis. Pattern Recogn Lett 27:861-874

9. Haissaguerre M, Jais P, Shah DC et al (1998) Spontaneous initiation of atrial fibrillation by ectopic beats originating in the pulmonary veins. N Engl J Med 339:659-666

10. Hunter RJ, Diab I, Thomas G et al (2009) Validation of a classification system to grade fractionation in atrial fibrillation and correlation with automated detection systems. Europace 11:1587-1596

11. Krueger MW, Schulze WH, Rhode KS, Razavi R, Seemann G, Dossel O (2013) Towards personalized clinical in silico modeling of atrial anatomy and electrophysiology. Med Biol Eng Comput 51:1251-1260

12. Landis JR, Koch GG (1977) The measurement of observer agreement for categorical data. Biometrics 33:159-174

13. Lau DH, Maesen B, Zeemering S et al (2015) Indices of bipolar complex fractionated atrial electrograms correlate poorly with each other and atrial fibrillation substrate complexity. Heart Rhythm 15:1415-1423

14. Lin YJ, Tai CT, Chang SL et al (2009) Efficacy of additional ablation of complex fractionated atrial electrograms for catheter ablation of nonparoxysmal atrial fibrillation. J Cardiovasc Electrophysiol 20:607-615

15. Monir G, Pollak SJ (2008) Consistency of the CFAE phenomena using custom software for automated detection of complex fractionated atrial electrograms (CFAEs) in the left atrium during atrial fibrillation. J Cardiovasc Electrophysiol 19:915-919

16. Nademanee K, McKenzie J, Kosar E et al (2004) A new approach for catheter ablation of atrial fibrillation: mapping of the electrophysiologic substrate. J Am Coll Cardiol 43:2044-2053

17. Narayan SM, Wright M, Derval N et al (2011) Classifying fractionated electrograms in human atrial fibrillation using monophasic action potentials and activation mapping: evidence for localized drivers, rate acceleration, and nonlocal signal etiologies. Heart Rhythm 8:244-253

18. Ng J, Borodyanskiy AI, Chang ET et al (2010) Measuring the complexity of atrial fibrillation electrograms. J Cardiovasc Electrophysiol 21:649-655

19. Oral H, Chugh A, Good E et al (2007) Radiofrequency catheter ablation of chronic atrial fibrillation guided by complex electrograms. Circulation 115:2606-2612

20. Oral H, Chugh A, Yoshida K et al (2009) A randomized assessment of the incremental role of ablation of complex fractionated atrial electrograms after antral pulmonary vein isolation for longlasting persistent atrial fibrillation. J Am Coll Cardiol 53:782-789

21. Porter M, Spear W, Akar JG et al (2008) Prospective study of atrial fibrillation termination during ablation guided by automated detection of fractionated electrograms. J Cardiovasc Electrophysiol 19:613-620

22. Redfearn DP, Simpson CS, Abdollah H, Baranchuk AM (2009) Temporo-spatial stability of complex fractionated atrial electrograms in two distinct and separate episodes of paroxysmal atrial fibrillation. Europace 11:1440-1444
23. Rostock T, Rotter M, Sanders P et al (2006) High-density activation mapping of fractionated electrograms in the atria of patients with paroxysmal atrial fibrillation. Heart Rhythm 3:27-34

24. Roux JF, Gojraty S, Bala R et al (2009) Effect of pulmonary vein isolation on the distribution of complex fractionated electrograms in humans. Heart Rhythm 6:156-160

25. Scherr D, Dalal D, Cheema A et al (2009) Long- and short-term temporal stability of complex fractionated atrial electrograms in human left atrium during atrial fibrillation. J Cardiovasc Electrophysiol 20:13-21

26. Sohal M, Choudhury R, Taghji P et al (2015) Is mapping of complex fractionated electrograms obsolete? Arrhythm Electrophysiol Rev 4:109-115

27. Tsai WC, Wang JH, Lin YJ et al (2012) Consistency of the automatic algorithm in detecting complex fractionated electrograms using an electroanatomical navigation system. Pacing Clin Electrophysiol 35:980-989

28. Tuan J, Jeilan M, Kundu S et al (2011) Regional fractionation and dominant frequency in persistent atrial fibrillation: effects of left atrial ablation and evidence of spatial relationship. Europace 13:1550-1556

29. Verma A, Jiang CY, Betts TR et al (2015) Approaches to catheter ablation for persistent atrial fibrillation. N Engl J Med 372:1812-1822

30. Verma A, Lakkireddy D, Wulffhart Z et al (2011) Relationship between complex fractionated electrograms (CFE) and dominant frequency (DF) sites and prospective assessment of adding dfguided ablation to pulmonary vein isolation in persistent atrial fibrillation (AF). J Cardiovasc Electrophysiol 22:1309-1316

31. Verma A, Mantovan R, Macle L et al (2010) Substrate and Trigger Ablation for Reduction of Atrial Fibrillation (STAR AF): a randomized, multicentre, international trial. Eur Heart J 31:1344-1356

32. Zlochiver S, Yamazaki M, Kalifa J, Berenfeld O (2008) Rotor meandering contributes to irregularity in electrograms during atrial fibrillation. Heart Rhythm 5:846-854

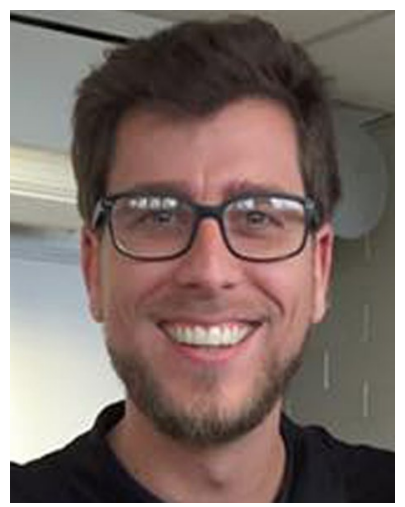

Mr. Tiago P. Almeida obtained a B.E. in Electrical Engineering at São Paulo State University in 2007 and M.Sc. in Biomedical Engineering at the University of Campinas in 2012. He has been doing his $\mathrm{PhD}$ studies at the University of Leicester.

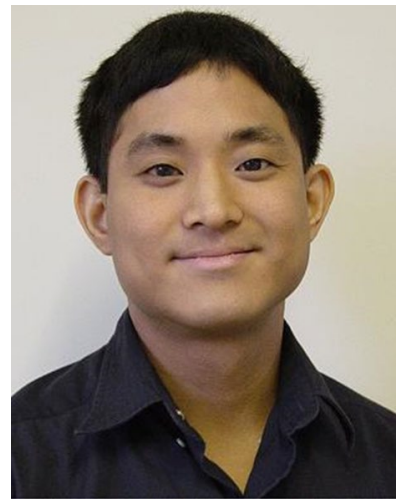

Dr. Gavin S. Chu graduated from the University of Cambridge in 2004. He is a Specialty Registrar in Cardiology and a Clinical Research Fellow in Cardiac Electrophysiology focusing on translational bioengineering in atrial fibrillation. 


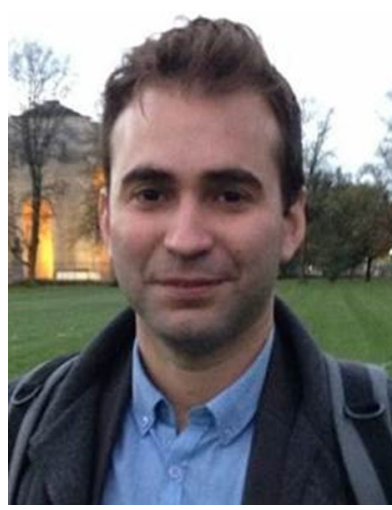

Dr. João L. Salinet has M.Sc. and Ph.D. in Biomedical Engineering from the University of Campinas and University of Leicester. Salinet is a lecturer in Bioengineering at Federal ABC University, and his research interests include cardiac arrhythmias mechanisms.

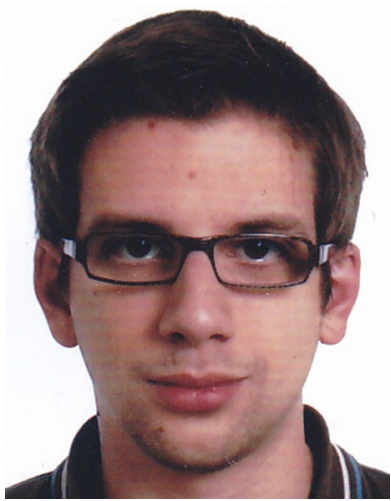

Dr. Frederique J. Vanheusden obtained B.Sc. and M.Sc. in Biomedicine at Hasselt University and M.Sc. in Molecular Medicine at Cranfield University. He obtained an Engineering Ph.D. at the University of Leicester.

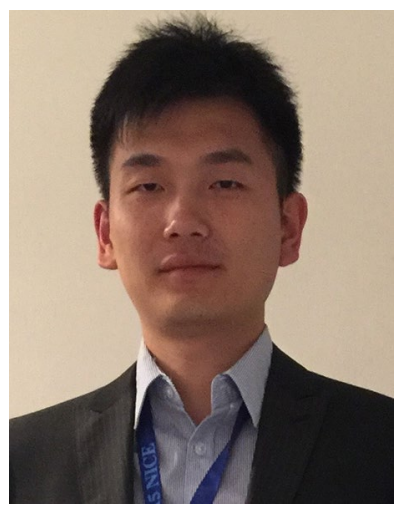

Mr. Xin Li was born in Mudanjiang, China, in 1989. He received B.Sc. in Electrical Information Engineering from the University of Science and Technology Beijing 2011, and M.Sc. in Electrical Electronic Engineering from the University of Leeds 2012.

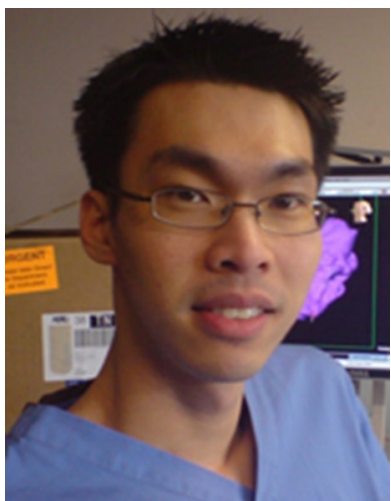

Dr. Jiun H. Tuan graduated from the University of Glasgow in 2002 and obtained his MD from the University of Leicester in 2012. He is currently a Consultant Cardiologist and Electrophysiologist at Hospital Lam Wah Ee, Malaysia.

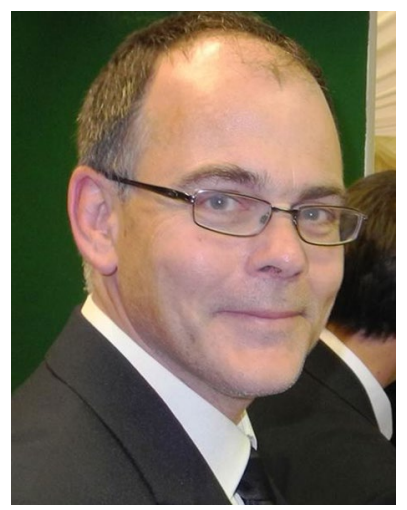

Dr. Peter J. Stafford has been training in cardiology and in electrophysiology for more than 20 years. He currently plays a lead role in the Regional Electrophysiology Service at Glenfield Hospital.

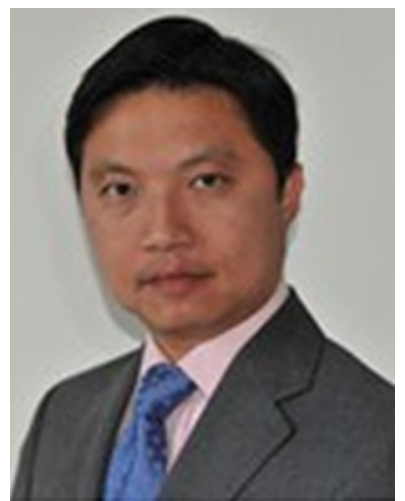

Prof. G. André Ng studied Medicine at the University of Glasgow where he obtained his $\mathrm{MBChB}$ and Ph.D. He was appointed as Senior Lecturer and Consultant Cardiologist and Electrophysiologist at the University Hospitals of Leicester in 2002, and was awarded Personal Chair of Cardiac Electrophysiology at the University of Leicester in 2013. He leads a team of talented researchers spanning preclinical, clinical, and biomedical engineering areas focused on cardiac

arrhythmias and electrophysiology.

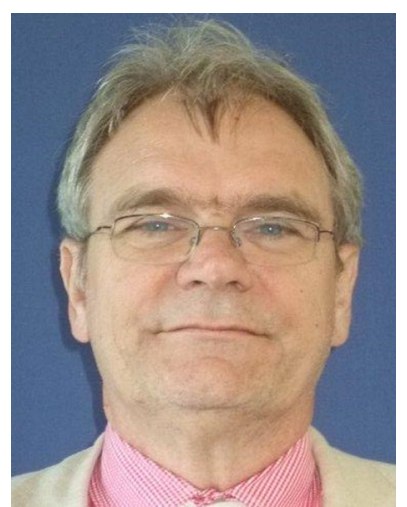

Dr. Fernando S. Schlindwein obtained an Electric Engineering degree in 1979 from the Federal University of Rio Grande do Sul, an M.Sc. in Biomedical Engineering from the Federal University of Rio de Janeiro, a Ph.D. in Biomedical Engineering from the Department of Surgery of the University of Leicester in 1990, and a D.Sc. in Biomedical Engineering from the Federal University of Rio de Janeiro in 1992. He joined the Department of Engineering at the University of Leicester as a lecturer in 1992. His main research interests are realtime signal processing and cardiac arrhythmias, especially the characterization and treatment for atrial fibrillation. 